\title{
Calculation of Intravascular Signal in Dynamic Contrast Enhanced-MRI Using Adaptive Complex Independent Component Analysis
}

\author{
Hatef Mehrabian*, Rajiv Chopra, and Anne L. Martel
}

\begin{abstract}
Assessing tumor response to therapy is a crucial step in personalized treatments. Pharmacokinetic (PK) modeling provides quantitative information about tumor perfusion and vascular permeability that are associated with prognostic factors. A fundamental step in most PK analyses is calculating the signal that is generated in the tumor vasculature. This signal is usually inseparable from the extravascular extracellular signal. It was shown previously using in vivo and phantom experiments that independent component analysis (ICA) is capable of calculating the intravascular time-intensity curve in dynamic contrast enhanced (DCE)-MRI. A novel adaptive complex independent component analysis (AC-ICA) technique is developed in this study to calculate the intravascular time-intensity curve and separate this signal from the DCE-MR images of tumors. The use of the complex-valued DCE-MRI images rather than the commonly used magnitude images satisfied the fundamental assumption of ICA, i.e., linear mixing of the sources. Using an adaptive cost function in ICA through estimating the probability distribution of the tumor vasculature at each iteration resulted in a more robust and accurate separation algorithm. The AC-ICA algorithm provided a better estimate for the intravascular time-intensity curve than the previous ICA-based method.
\end{abstract}

A simulation study was also developed in this study to realistically simulate DCE-MRI data of a leaky tissue mimicking phantom. The passage of the MR contrast agent through the leaky phantom was modeled with finite element analysis using a diffusion model. Once the distribution of the contrast agent in the imaging field of view was calculated, DCE-MRI data was generated by solving the Bloch equation for each voxel at each time point.

The intravascular time-intensity curve calculation results were compared to the previously proposed ICA-based intravascular time-intensity curve calculation method that applied ICA to the magnitude of the DCE-MRI data (Mag-ICA) using both simulated and experimental tissue mimicking phantoms. The AC-ICA demonstrated superior performance compared to the Mag-ICA method. AC-ICA provided more accurate estimate of intravascular time-intensity curve, having smaller error between

Manuscript received November 21, 2012; accepted December 05, 2012. Date of publication December 12, 2012; date of current version March 29, 2013. This work was supported by the Natural Sciences and Engineering Research Council of Canada (NSERC). Asterisk indicates corresponding author.

${ }^{*} \mathrm{H}$. Mehrabian is with the Department of Medical Biophysics, University of Toronto, Toronto, ON, M5G 2M9 Canada and also with the Physical Sciences, Sunnybrook Research Institute, Toronto, ON, M4N 3M5 Canada (e-mail: hatef. mehrabian@sri.utoronto.ca).

R. Chopra is with the Department of Radiology, University of Texas Southwestern Medical Center, Dallas, TX 75390 USA.

A. L. Martel is with the Department of Medical Biophysics, University of Toronto, Toronto, ON, M5G 2M9 Canada and also with the Physical Sciences, Sunnybrook Research Institute, Toronto, ON, M4N 3M5 Canada.

Color versions of one or more of the figures in this paper are available online at http://ieeexplore.ieee.org.

Digital Object Identifier 10.1109/TMI.2012.2233747 the calculated and actual intravascular time-intensity curves compared to the Mag-ICA.

Furthermore, it showed higher robustness in dealing with datasets with different resolution by providing smaller variation between the results of each datasets and having smaller difference between the intravascular time-intensity curves of various resolutions. Thus, AC-ICA has the potential to be used as the intravascular time-intensity curve calculation method in PK analysis and could lead to more accurate PK analysis for tumors.

Index Terms-Adaptive complex independent component analysis (AC-ICA), arterial input function (AIF), intravascular signal intensity, pharmacokinetic modeling.

\section{INTRODUCTION}

D ERSONALIZED therapy is becoming more viable as our understanding of cancer biology and treatment options improve. Considering the high cost and specificity of these treatments, selection of the proper patient population and rapid assessment of their therapeutic response is of upmost importance [1], [2] and has to be improved. However, currently used approaches to assess tumor response to therapy, i.e., tumor size measurement through response evaluation criteria in solid tumors (RECIST) and serum marker evaluation, have several limitations. Many advanced treatments, although effective, do not affect the tumor size and most tumors do not produce enough biomarkers to be used in their evaluation. Thus, there is increasing interest in developing novel metrics using functional imaging techniques such as dynamic contrast enhanced (DCE)-MRI or positron emission tomography (PET) [2], [3].

Dynamic contrast enhanced-MR imaging of a tumor followed by pharmacokinetic (PK) modeling to assess and quantify contrast agent kinetics is one of the main approaches to assess tumor response to therapy. Such a DCE-MRI study involves an intravenous injection of a bolus of a low molecular weight contrast agent, e.g., Gadolinium (Gd)-DTPA, followed by repeated imaging of the tumor area to track the passage of the bolus through the tumor vasculature. The PK model provides a way to quantify the leakage of the contrast agent from the tumor vasculature or plasma volume into the extravascular extracellular space (EES). Such a model provides information about the tumor permeability and blood volume that have been shown to be related to prognostic factors [4] and thus can be used in evaluation of anti-angiogenic and anti-vascular therapies [5].

There are several PK models such as the Tofts and the extended Tofts models that assume instantaneous mixing of the blood plasma and contrast agent as it arrives in the tumor, and 
the adiabatic approximation to the tissue homogeneity (AATH) model that assumes the contrast concentration in the EES is defined as a function of transit time and the distance along the capillary [6], [7]. Determining the contrast agent concentration in the intravascular space is a fundamental step in most PK models of tumor tissues [8]. Due to the heterogeneity of tumor vasculature, low resolution of clinical images and partial volume effect, there is no direct method to separate the signal that is generated in the tumor vasculature from the EES signal and thus it is extremely difficult to determine the intravascular contrast concentration in the tumor [9].

Thus, it is common to estimate the intravascular contrast concentration using an arterial input function (AIF). This AIF is measured using the concentration in a major artery that is adjacent to the tumor and is feeding the tumor [10]. Other AIF approximation methods include, using a standard AIF [11], reference tissue based [12] or population average AIF [13]. Since all of these approaches try to approximate the AIF outside of the tumor, they introduce error to the system and its correction steps make the system of equations complicated. For instance these methods, either assume that there is no delay between the arrival of contrast agent in the AIF and in the tumor, or they introduce a delay parameter in the model making the system of equations more complex. The standard AIF can be assumed to take a bi-exponential form; alternatively a population average AIF can be used. Both of these methods fail to account for the intra-subject variability of the AIF. The reference tissue method approximates the AIF in a normal tissue (usually muscle tissue) by assuming that the PK model parameters of the normal tissue are known from the literature [12]. It was shown in [14] that these parameters vary between subjects and using literature values for normal tissue does not provide accurate results. In studies where the AIF is calculated in a major artery it is assumed that the selected artery is feeding the tumor and that no other artery is supplying blood to the tumor [13], [15]. Such an AIF is unable to capture the early phases of the passage of the contrast agent through the tumor vasculature and thus a reference region based correction method has been introduced in [14]. The effect of adding more parameters to the PK model was studied in [16] and it was concluded that although these parameters make the model more accurate in theory, due to increased complexity and instability of the system of equations, they are unable to improve the results of PK analysis in practice.

Each voxel in an MR image is partially occupied by blood vessels or the intravascular space and the rest is the extravascular structures. Consequently, the MR signal in each voxel is the sum of the signal that is generated in the intravascular space and the signal from the extravascular space. The low molecular weight contrast agents used in DCE-MRI studies do not enter the cells, and thus the signal can be assumed to be generated in the intravascular and the extravascular extracellular spaces. In previous work [15], [17] we introduced an independent component analysis (ICA)-based method to calculate intravascular time-intensity curve in DCE-MRI studies inside the tumor. This method assumed that the intravascular and extravascular extracellular components of the DCE-MRI data of the tumor are spatially independent and are linearly mixed to form the MR images in each voxel. This method was shown to have good performance in calculating the intravascular time-intensity curve and separating it from the data and its results were validated against tissue-mimicking phantoms and contrast enhanced ultrasound imaging of the tumor vasculature in vivo. The method performed well in spite of the fact that the linear mixture assumption, which is a fundamental assumption for ICA, was violated since processing was carried out on the magnitude of the MRI data. The original MRI signal in each voxel that is a linear combination of the intravascular and extravascular extracellular signals is complex-valued. However, the signals of these two spaces (intravascular and extravascular extracellular spaces) usually are not in-phase and their signals will partially cancel each other. Thus the magnitude of the sum of their signals would not be equal to the sum of the magnitudes of the signal of the two spaces, which violates the linearity assumption in ICA. This problem was tackled by using a small echo time (TE) to minimize intra-voxel de-phasing of the spins.

In addition, in that work [15], [17], the probability distribution functions of the intravascular and extravascular extracellular spaces were not estimated and, as is common in ICA algorithms, a fixed cost function which does not account for the intra-subject variability between the spatial distributions of tumor vasculature was used instead.

A more rigorous way of addressing these problems would be to analyze the complex data rather than the magnitude data. An adaptive complex ICA (AC-ICA) method is introduced here which uses the complex-valued MRI data where the linear mixture assumption of ICA is satisfied. The proposed AC-ICA method also determines the ICA cost function based on the distribution of the vasculature through an expectation maximization (EM) procedure by performing online density estimation at each iteration. The performance of the AC-ICA method is evaluated using simulation and experimental tissue-mimicking phantoms and the results are compared to the previously introduced ICA-based method in [15], [17], that applied ICA to the magnitude of the DCE-MRI data and used a fixed cost function (Mag-ICA).

The structure of this paper is as follows: the theory and methods section briefly explains the Mag-ICA method. It also explains the complex ICA approach that is used in the AC-ICA followed by the expectation maximization procedure that is used to estimate the distribution of the tumor vasculature and find its proper cost function. The simulation phantom is then explained and the Bloch equation simulation that is used to generate the simulated DCE-MRI dataset is described. This section also describes the experimental tissue mimicking phantom that was constructed and explains its DCE-MRI data acquisition procedure. The results section gives the results of applying both AC-ICA and Mag-ICA methods to simulation and experimental phantom data. It also analyzes the robustness of both methods and the reproducibility of their results. The discussions section explains the main challenges of the AC-ICA method and its main differences with Mag-ICA.

\section{THEORY AND METHODS}

\section{A. Independent Component Analysis}

ICA is a statistical signal processing method that attempts to split a dataset into its underlying features, assuming these features are statistically independent and without assuming any 
knowledge of the mixing coefficients [18]. In this article we will use capital bold letter for 2-D matrices, lowercase bold letters for column vectors, capital or lowercase letters (not bold) for scalars and, bold italic letters for functions. When the features are mixed linearly, the ICA model is expressed as

$$
\mathbf{Z}=\mathbf{A} \mathbf{S}
$$

where $\boldsymbol{Z}=\left[\mathbf{z}_{1}, \mathbf{z}_{2}, \ldots, \mathbf{z}_{\mathbf{N}}\right]^{\mathbf{T}}$ represents the time-series dataset which in this study is the complex-valued DCE-MRI dataset of a tumor or a tissue-mimicking phantom (observed mixtures) and $\mathrm{N}$ is the number of time points in the DCE-MRI sequence, $\mathbf{S}=\left[\mathbf{s}_{1}, \mathbf{s}_{2}, \ldots, \mathbf{s}_{\mathbf{M}}\right]^{\mathbf{T}}$ is a matrix containing the M structures that are known as independent components or ICs (usually M $\leq \mathrm{N}$ ) and in this study these are the images representing the intravascular and extravascular extracellular spaces of the tumor tissue. Although we assume that there are only two spaces in our model (intravascular and extravascular extracellular), since ICA makes no assumption about the spatial distribution of these spaces, it might split each space into several components. In practice more than two ICs are required to achieve accurate separation. $\mathbf{A} \in \mathbb{R}^{\mathrm{N} \times \mathrm{M}}$ is the mixing matrix whose columns represent the contrast uptake curves of the intravascular and extravascular extracellular compartments. Having the observed mixture signals $\mathbf{Z}$, the ICA method attempts to estimate the underlying features (independent components) $\mathbf{S}$ and the mixing matrix $\mathbf{A}$. This is achieved by finding an unmixing matrix $\mathbf{W} \in \mathbb{R}^{\mathrm{M} \times \mathrm{N}}$ and estimating the IC matrix $\mathbf{Y}=\left[\mathbf{y}_{\mathbf{1}}, \mathbf{y}_{\mathbf{2}}, \ldots, \mathbf{y}_{\mathbf{M}}\right]^{\mathbf{T}}$ such that

$$
\mathbf{Y}=\mathbf{W Z}
$$

where the rows of $\mathbf{Y}$ are statistically independent and have zero mean, and unit variance, i.e., $\boldsymbol{E}\left\{\mathbf{Y} \mathbf{Y}^{H}\right\}=\boldsymbol{I}$, where $H$ is Hermitian transform and $\boldsymbol{E}\{$.$\} is the expectation operator. The IC's$ can be recovered up to an arbitrary scaling and permutation [18].

Phase Shifting of DCE-MRI Data for ICA: Assume a 2-D matrix $\mathbf{Q} \in \mathbb{R}^{\mathrm{N}_{\mathrm{r}} \times \mathrm{N}_{\mathrm{c}}}$, where $\mathrm{N}_{\mathrm{r}}$ represents the number of rows and $\mathrm{N}_{\mathrm{c}}$ represents the number of columns of $\mathrm{Q}$, is being used as the input to Fourier transform (FT) to generate the MRI data. FT assumes that the zero frequency point is the initial point of the signal located at the first row and the first column of Q. However in MRI data acquisition in k-space, the zero frequency point is stored at the center of the k-space (located at the row $\mathrm{N}_{\mathrm{r}} / 2$ and column $\mathrm{N}_{\mathrm{c}} / 2$ ) and then higher frequency elements are stored around this center point which is not the arrangement that is expected by FT.

This rearrangement of the $\mathrm{k}$-space values corresponds to displacement of data located at row $n$ and column $m$ with $\mathrm{N}_{\mathrm{r}} / 2$ rows and $\mathrm{N}_{\mathrm{c}} / 2$ columns in $\mathrm{k}$-space. This is equivalent to a phase shift of $\mathrm{e}^{i 2 \pi \mathrm{n}\left(\mathrm{N}_{\mathrm{r}} / 2\right) / \mathrm{N}_{\mathrm{r}}} \mathrm{e}^{i 2 \pi \mathrm{m}\left(\mathrm{N}_{\mathrm{c}} / 2\right) / \mathrm{N}_{\mathrm{c}}}=(-1)^{\mathrm{n}+\mathrm{m}}$ which translates to a sign alteration of every other point. This phase shift has to be corrected by changing the sign of every other point in complex DCE-MRI data. The phase shift has no effect when using the magnitude of the MRI data but affects the analysis when the complex data is being used. If this phase shift is not corrected prior to the application of ICA, then the values of neighboring voxels cancel out, in particular when computing the mean and covariance of the signal.

Magnitude ICA (Mag-ICA): According to the central limit theorem, the distribution of a sum of independent random variables with finite support probability density functions (pdf) tends towards a Gaussian distribution [19]. Thus, by maximizing the non-Gaussianity of the estimated components, the independent components can be identified. In an information theoretic framework, one way of measuring non-Gaussianity of a real-valued random variable $(\mathbf{y})$ is to measure its Negentropy [18] given by

$$
\boldsymbol{J}_{\text {negentropy }}(\mathbf{y})=\boldsymbol{H}\left(\mathbf{y}_{\text {gauss }}\right)-\boldsymbol{H}(\mathbf{y})
$$

where $\boldsymbol{H}(\mathbf{y})=-\boldsymbol{E}\{\log (\boldsymbol{p}(\mathbf{y}))\}$ is the differential entropy of $\mathbf{y}, \boldsymbol{p}($.$) is the pdf, and \mathbf{y}_{\text {gauss }}$ is a Gaussian random variable with the same variance as $\mathbf{y}$. Since the probability distributions of the ICs are not known, it is common in ICA algorithms to maximize the Negentropy by maximizing the following equation:

$$
\boldsymbol{J}_{\text {negentropy }}(\mathbf{y})=\boldsymbol{E}\{\boldsymbol{G}(\mathbf{y})\}
$$

where $\boldsymbol{G}($.$) is a nonquadratic nonlinearity function. The$ Mag-ICA method used skewness function as its nonlinearity $\boldsymbol{G}(\mathbf{y})=\mathbf{y}^{3}$, and used a Newton like method to maximize the Negentropy [15], [17].

Complex ICA: The Negentropy for the complex-valued random variable was defined using the joint distribution of its real and imaginary parts, i.e., $\boldsymbol{p}\left(\mathbf{z}^{R}, \mathbf{z}^{I}\right)$ in [20]

$$
\boldsymbol{J}_{\text {negentropy }}\left(\mathbf{z}^{R}, \mathbf{z}^{I}\right)=\boldsymbol{H}\left(\mathbf{z}_{\text {gauss }}^{R}, \mathbf{z}_{\text {gauss }}^{I}\right)-\boldsymbol{H}\left(\mathbf{z}^{R}, \mathbf{z}^{I}\right)
$$

where $\mathbf{z}^{R}$ and $\mathbf{z}^{I}$ are the real and imaginary parts of $\mathbf{z}$ and $\boldsymbol{H}\left(\mathbf{z}^{R}, \mathbf{z}^{I}\right)=-\boldsymbol{E}\left\{\log \left(\boldsymbol{p}\left(\mathbf{z}^{R}, \mathbf{z}^{I}\right)\right)\right\}$ is the bi-variate entropy. The complex Negentropy is always positive, and for a fixed covariance of $\left[\mathbf{z}^{R}, \mathbf{z}^{I}\right]^{T}$, the bi-variate entropy has its largest value for $\left[\mathbf{z}_{\text {gauss }}^{R}, \mathbf{z}_{\text {gauss }}^{I}\right]$. Thus, maximum non-Gaussianity is achieved by minimizing the bi-variate entropy $\left(\boldsymbol{H}\left(\mathbf{z}^{R}, \mathbf{z}^{I}\right)\right)$.

Assuming $\boldsymbol{p}\left(\mathbf{z}^{R}, \mathbf{z}^{I}\right)=\exp (-\boldsymbol{G}(\mathbf{z}))$, the cost function for complex maximization of negentropy (CMN) is given by [20]

$$
\boldsymbol{J}_{\text {negentropy }}(\mathbf{w})=\boldsymbol{E}\{\boldsymbol{G}(\mathbf{y})\}
$$

where $\mathbf{y}=\mathbf{w}^{H} \mathbf{Z}$, and $\boldsymbol{G}(\mathbf{y})=|\boldsymbol{C}(\mathbf{y})|^{2}$ in which $\boldsymbol{C}($.$) is the$ nonlinearity function for ICA. The expression for the maximization is given as [20]

$$
\mathbf{w}_{\text {optimum }}=\underset{\|\mathbf{w}\|=1}{\operatorname{argmax}}\left\{\boldsymbol{E}\left\{\left|\boldsymbol{C}\left(\mathbf{w}^{H} \mathbf{Z}\right)\right|^{2}\right\}\right\} .
$$

This constrained optimization is solved using a quasi-Newton method [20]. The Lagrangian function is

$$
\boldsymbol{L}(\mathbf{w}, \lambda)=\boldsymbol{J}_{\text {negentropy }}(\mathbf{w})+\lambda\left(\mathbf{w}^{H} \mathbf{w}-1\right)
$$

where $\lambda$ is the Lagrange multiplier. Using the complex Newton update defined in [21], the optimization update rule becomes [20]

$$
\mathbf{w}^{(i+1)}=\mathbf{w}^{(i)}-\left(\boldsymbol{H}_{J}-\lambda \mathbf{I}\right)^{-1}\left(\nabla_{J}^{*}+\lambda \mathbf{w}^{(i)}\right)
$$

where $\boldsymbol{H}_{J}$ and $\boldsymbol{\nabla}_{J}$ are the complex Hessian and the complex gradient matrices of $\boldsymbol{J}$, respectively. The fixed point update for $\mathbf{w}$ was derived from (1) in [20] as the following equation:

$$
\begin{aligned}
\mathbf{w}_{(i+1)}= & -\boldsymbol{E}\left\{\boldsymbol{C}^{*}\left(\mathbf{w}_{(i)}^{H} \mathbf{Z}\right) \boldsymbol{C}^{\prime}\left(\mathbf{w}_{(i)}^{H} \mathbf{Z}\right) \mathbf{Z}\right\} \\
& +\boldsymbol{E}\left\{\boldsymbol{C}^{\prime}\left(\mathbf{w}_{(i)}^{H} \mathbf{Z}\right) \boldsymbol{C}^{\prime *}\left(\mathbf{w}_{(i)}^{H} \mathbf{Z}\right)\right\} \mathbf{w}_{(i)} \\
& +\boldsymbol{E}\left\{\mathbf{Z} \mathbf{Z}^{T}\right\} \boldsymbol{E}\left\{\boldsymbol{C}^{*}\left(\mathbf{w}_{(i)}^{H} \mathbf{Z}\right) \boldsymbol{C}^{\prime \prime}\left(\mathbf{w}_{(i)}^{H} \mathbf{Z}\right)\right\} \mathbf{w}_{(i)}^{*}
\end{aligned}
$$

where $C^{*}, C^{\prime}$, and $C^{\prime \prime}$ are the complex conjugate, the first derivative and the second derivative of $\boldsymbol{C}$, respectively. 


\section{B. Adaptive Complex ICA (AC-ICA)}

The optimal non-Gaussianity in an information theoretic framework is the logarithm of the joint probability density function of the source that is being estimated, i.e., $\boldsymbol{G}_{\text {optimal }}(\mathbf{z})=-\log \left(\boldsymbol{p}\left(\mathbf{z}^{R}, \mathbf{z}^{I}\right)\right)$. However, the joint probability density of the source is not known in ICA and thus has to be estimated.

The generalized Gaussian distribution (GGD) is given as

$$
\boldsymbol{g}(\mathbf{y} ; \alpha, \beta)=\frac{\beta}{2 \alpha \boldsymbol{\Gamma}\left(\frac{1}{\beta}\right)} \exp \left(-\frac{|\mathbf{y}|^{\beta}}{\alpha^{\beta}}\right)
$$

where $\boldsymbol{\Gamma}($.$) is the gamma function defined as \boldsymbol{\Gamma}(\mathbf{x})=$ $\int_{0}^{\infty} e^{-t} t^{\mathbf{x}-1} d t$, and $\beta$ and $\alpha$ are the model parameters. The GGD distribution covers a wide range of distributions [22] and has been used in modeling various physical phenomena [23]-[25].

GGD was introduced in [22] as a cost function for ICA. We have observed that distribution of the real and imaginary parts of the MR images of each compartment (source) fits well into the GGD formulation. Assuming the linear mixture model holds for the MRI data in complex domain we modeled the MR image as a sum of a number of functions with GGD distributions.

Using an expectation maximization framework (explained in the next section) the parameters of these GGD distributions are found at each iteration and the GGD distribution with the highest membership probability is used to derive the nonlinearity in our ICA algorithm. Substituting the parameters $(\alpha, \beta)$ of the selected GGD distribution in (2) and using the relationship between the ICA nonlinearity and the pdf of the sources, i.e., $\boldsymbol{p}\left(\mathbf{z}^{R}, \mathbf{z}^{I}\right)=\exp \left(-|\boldsymbol{C}(\mathbf{z})|^{2}\right)$, the nonlinearity is defined as

$$
\boldsymbol{C}(\mathbf{z})=\left(\frac{\mathbf{z}}{\alpha}\right)^{\frac{\beta}{2}}
$$

\section{Expectation Maximization}

We developed an expectation maximization framework to calculate the parameters of the adaptive probability distribution by modeling the pdf of the estimated component as a mixture of a number of GGD distributions at each iteration. Assuming the probability density function of the estimated component $\mathbf{y}$ at each iteration, i.e., $\boldsymbol{f}(\mathbf{y}, \boldsymbol{\Theta})$, is comprised of $\mathrm{k}$ random variables with GGD distributions of the form $\boldsymbol{g}\left(\mathbf{y} ; \alpha_{\mathrm{k}}, \beta_{\mathrm{k}}\right)=\left(\beta_{\mathrm{k}} / 2 \alpha_{\mathrm{k}} \boldsymbol{\Gamma}\left(1 / \beta_{\mathrm{k}}\right)\right) \exp \left(-|\mathbf{y}|^{\beta_{\mathrm{k}}} / \alpha_{\mathrm{k}}^{\beta_{k}}\right)$, and each GGD contributes to formation of the pdf of $\mathbf{y}$ with a membership probability $\mathrm{p}_{\mathrm{k}}$ we have

$$
\boldsymbol{f}(\mathbf{y}, \boldsymbol{\Theta})=\sum_{k=1}^{\mathrm{K}} \mathrm{p}_{\mathrm{k}} \boldsymbol{g}\left(\mathbf{y} ; \alpha_{\mathrm{k}}, \beta_{\mathrm{k}}\right)
$$

where $\boldsymbol{\Theta}=\left[\boldsymbol{\theta}_{1}, \boldsymbol{\theta}_{2}, \ldots, \boldsymbol{\theta}_{\mathrm{k}}\right]=\left[\left(\mathrm{p}_{1}, \alpha_{1}, \beta_{1}\right), \ldots,\left(\mathrm{p}_{\mathrm{k}}, \alpha_{\mathrm{k}}, \beta_{\mathrm{k}}\right)\right]$ is the parameter space. $\boldsymbol{f}(\mathbf{y}, \boldsymbol{\Theta})$ is a probability density function, thus

$$
\begin{aligned}
\int_{R^{2}} \boldsymbol{f}(\mathbf{y}, \boldsymbol{\Theta}) & =\int_{R^{2}} \sum_{\mathrm{k}=1}^{\mathrm{K}} p_{\mathrm{k}} \boldsymbol{g}\left(\mathbf{y} ; \alpha_{\mathrm{k}}, \beta_{\mathrm{k}}\right) \\
& =\sum_{\mathrm{k}=1}^{\mathrm{K}} \mathrm{p}_{\mathrm{k}} \int_{R^{2}} \boldsymbol{g}\left(\mathbf{y} ; \alpha_{\mathrm{k}}, \beta_{\mathrm{k}}\right)=1
\end{aligned}
$$

$\boldsymbol{g}\left(\mathbf{y} ; \alpha_{\mathrm{k}}, \beta_{\mathrm{k}}\right)$ is also a probability density function, therefore $\int_{R^{2}} \boldsymbol{g}\left(\mathbf{y} ; \alpha_{\mathrm{k}}, \beta_{\mathrm{k}}\right)=1$, and thus

$$
\int_{R^{2}} \boldsymbol{f}(\mathbf{y}, \boldsymbol{\Theta})=\sum_{\mathrm{k}=1}^{\mathrm{K}} \mathrm{p}_{\mathrm{k}}=1
$$

and since $p_{k}$ are probabilities, they are nonnegative $\left(p_{k} \geq 0\right)$.

The maximum Log-likelihood estimate can be formulated as

$$
\begin{aligned}
\boldsymbol{\Lambda}(\mathbf{y}, \boldsymbol{\Theta})=\log \prod_{\mathrm{n}=1}^{\mathrm{N}} \boldsymbol{f}(\mathbf{y}, \boldsymbol{\Theta}) & =\log \prod_{\mathrm{n}=1}^{\mathrm{N}} \sum_{\mathrm{k}=1}^{\mathrm{K}} \mathrm{p}_{\mathrm{k}} \boldsymbol{g}\left(\mathbf{y} ; \alpha_{\mathrm{k}}, \beta_{\mathrm{k}}\right) \\
& =\sum_{\mathrm{n}=1}^{\mathrm{N}} \log \sum_{\mathrm{k}=1}^{\mathrm{K}} \mathrm{p}_{\mathrm{k}} \boldsymbol{g}\left(\mathrm{y} ; \alpha_{\mathrm{k}}, \beta_{\mathrm{k}}\right)
\end{aligned}
$$

where $\mathrm{N}$ represents the number of samples in $\mathbf{y}$. The maximum likelihood estimation is formulated as

$$
\widehat{\boldsymbol{\Theta}}=\underset{\boldsymbol{\Theta}}{\operatorname{argmax}}\{\boldsymbol{\Lambda}(\mathbf{y}, \boldsymbol{\Theta})\}
$$

defining $\boldsymbol{q}(\mathrm{k}, \mathrm{n})=\mathrm{p}_{\mathrm{k}} \boldsymbol{g}\left(\mathrm{y}_{\mathrm{n}} ; \alpha_{\mathrm{k}}, \beta_{\mathrm{k}}\right)$, results in the following conditional probabilities that are called membership probabilities:

$$
\boldsymbol{p}(\mathrm{k} \mid \mathrm{n})=\frac{\boldsymbol{q}(\mathrm{k}, \mathrm{n})}{\Sigma_{\mathrm{m}=1}^{\mathrm{K}} \boldsymbol{q}(\mathrm{m}, \mathrm{n})}
$$

using Jensen's inequalities [26], i.e., $\log \sum_{\mathrm{k}=1}^{\mathrm{K}} b_{\mathrm{k}} \geq$ $\sum_{\mathrm{k}=1}^{\mathrm{K}} a_{\mathrm{k}} \log \left(b_{\mathrm{k}} / a_{\mathrm{k}}\right)$, the Log-likelihood at each iteration $(i)$ can be expressed as

$$
\begin{aligned}
\boldsymbol{\Lambda}(\mathbf{y}, \boldsymbol{\Theta}) & =\sum_{\mathrm{n}=1}^{\mathrm{N}} \log \sum_{\mathrm{k}=1}^{\mathrm{K}} \boldsymbol{q}(\mathrm{k}, \mathrm{n}) \\
& \geq \sum_{\mathrm{n}=1}^{\mathrm{N}} \sum_{\mathrm{k}=1}^{\mathrm{K}} \boldsymbol{p}^{(i)}(\mathrm{k} \mid \mathrm{n}) \log \frac{\boldsymbol{q}(\mathrm{k}, \mathrm{n})}{\boldsymbol{p}^{(i)}(\mathrm{k} \mid \mathrm{n})}=\boldsymbol{b}_{(i)}(\boldsymbol{\Theta})
\end{aligned}
$$

where $\boldsymbol{b}_{(i)}(\boldsymbol{\Theta})$ is the lower bound for the Log-likelihood function. Thus, to calculate the maximum Log-likelihood estimator we need to maximize its lower bound, $\boldsymbol{b}_{(i)}(\boldsymbol{\Theta})$ iteratively

$$
\begin{aligned}
\boldsymbol{b}_{(i)}(\boldsymbol{\Theta})= & \sum_{\mathrm{n}=1}^{\mathrm{N}} \sum_{\mathrm{k}=1}^{\mathrm{K}} \boldsymbol{p}^{(i)}(\mathrm{k} \mid \mathrm{n}) \log \boldsymbol{q}(\mathrm{k}, \mathrm{n}) \\
& -\sum_{\mathrm{n}=1}^{\mathrm{N}} \sum_{\mathrm{k}=1}^{\mathrm{K}} \boldsymbol{p}^{(i)}(\mathrm{k} \mid \mathrm{n}) \log \boldsymbol{p}^{(i)}(\mathrm{k} \mid \mathrm{n}) .
\end{aligned}
$$

The second term of the right-hand side of (3) is a constant as it is calculated from the old values. Thus, the maximization problem is simplified to

$$
\begin{aligned}
\widehat{\boldsymbol{\Theta}} & =\max _{\boldsymbol{\Theta}}\left\{\boldsymbol{b}_{(i)}(\boldsymbol{\Theta})\right\} \\
& =\max _{\mathrm{p}_{\mathrm{k}}, \alpha_{\mathrm{k}}, \beta_{\mathrm{k}}}\left\{\sum_{\mathrm{n}=1}^{\mathrm{N}} \sum_{\mathrm{k}=1}^{\mathrm{K}} \boldsymbol{p}^{(i)}(\mathrm{k} \mid \mathrm{n}) \log \boldsymbol{q}(\mathrm{k}, \mathrm{n})\right\} .
\end{aligned}
$$

The probabilities $\boldsymbol{p}(\mathrm{k} \mid \mathrm{n})$ can be calculated at each iteration $(i)$ using

$$
\begin{aligned}
& \boldsymbol{p}^{(i)}(\mathrm{k} \mid \mathrm{n})=\frac{\mathrm{p}_{\mathrm{k}}^{(i)} \boldsymbol{g}\left(\mathrm{y}_{\mathrm{n}} ; \alpha_{\mathrm{k}}^{(i)}, \beta_{\mathrm{k}}^{(i)}\right)}{\sum_{\mathrm{m}=1}^{\mathrm{K}} \mathrm{p}_{\mathrm{m}}^{(i)} \boldsymbol{g}\left(\mathrm{y}_{\mathrm{n}} ; \alpha_{\mathrm{m}}^{(i)}, \beta_{\mathrm{m}}^{(i)}\right)} \\
& \& \sum_{\mathrm{k}=1}^{\mathrm{K}} \boldsymbol{p}^{(i)}(\mathrm{k} \mid \mathrm{n})=1 .
\end{aligned}
$$


Setting the derivative of $\boldsymbol{b}_{(i)}(\boldsymbol{\Theta})$ with respect to $\alpha_{\mathrm{k}}$ equal to zero we have

$$
\frac{\partial[\boldsymbol{b}(\boldsymbol{\Theta})]}{\partial \alpha_{\mathrm{k}}}=\sum_{n=1}^{\mathrm{N}} \boldsymbol{p}(\mathrm{k} \mid \mathrm{n}) \frac{\partial\left[\log \left(\mathrm{p}_{\mathrm{k}} \boldsymbol{g}\left(\mathrm{y}_{\mathrm{n}} ; \alpha_{\mathrm{k}}, \beta_{\mathrm{k}}\right)\right)\right]}{\partial \alpha_{\mathrm{k}}}=0
$$

which results in the following expression for $\alpha_{\mathrm{k}}^{(i)}$ at each iteration

$$
\alpha_{k}^{(i)}=\left[\frac{\beta_{k}^{(i)} \sum_{n=1}^{\mathrm{N}}\left(\boldsymbol{p}^{(i)}(\mathrm{k} \mid \mathrm{n})\left|\mathrm{y}_{n}\right|^{\beta_{k}^{(i)}}\right)}{\mathrm{N}}\right]^{\frac{1}{\beta_{k}^{(i)}}} .
$$

Setting the derivative of $\boldsymbol{b}_{(i)}(\boldsymbol{\Theta})$ with respect to $\beta_{k}$ equal to zero we have

$$
\frac{\partial[\boldsymbol{b}(\boldsymbol{\Theta})]}{\partial \beta_{k}}=\sum_{n=1}^{\mathrm{N}} \boldsymbol{p}(\mathrm{k} \mid \mathrm{n}) \frac{\partial\left[\log \left(\mathrm{p}_{k} \boldsymbol{g}\left(\mathrm{y}_{\mathrm{n}} ; \alpha_{\mathrm{k}}, \beta_{\mathrm{k}}\right)\right)\right]}{\partial \beta_{k}}=0 .
$$

This equation results in (6) that will be used to calculate $\beta_{\mathrm{k}}^{(\mathrm{i})}$ at each iteration

$$
\begin{aligned}
\frac{1}{\beta_{\mathrm{k}}^{(\mathrm{i})}}(1+ & \left.\frac{1}{\beta_{\mathrm{k}}^{(\mathrm{i})}} \boldsymbol{\psi}\left(\frac{1}{\beta_{\mathrm{k}}^{(\mathrm{i})}}\right)\right) \sum_{\mathrm{n}=1}^{\mathrm{N}} \boldsymbol{p}^{(\mathrm{i})}(\mathrm{k} \mid \mathrm{n}) \\
& \quad-\sum_{\mathrm{n}=1}^{\mathrm{N}} \boldsymbol{p}^{(\mathrm{i})}(\mathrm{k} \mid \mathrm{n})\left(\frac{\left|\mathrm{y}_{\mathrm{n}}\right|}{\alpha_{\mathrm{k}}^{(\mathrm{i})}}\right)^{\beta_{\mathrm{k}}^{(\mathrm{i})}} \log \left(\frac{\left|\mathrm{y}_{\mathrm{n}}\right|}{\alpha_{\mathrm{k}}^{(\mathrm{i})}}\right)=0
\end{aligned}
$$

where $\boldsymbol{\psi}(\mathbf{x})=d(\log (\boldsymbol{\Gamma}(x))) / d \mathbf{x}$, is the polygamma function. This equation can be solved using any optimization algorithm. We used the fzero function of MATLAB (The MathWorks Inc., Natick, MA, USA) software to find the value of $\beta_{\mathrm{k}}^{(\mathrm{i})}$ at each iteration.

These three equations are solved at each iteration and the parameter set $\left(\mathrm{p}_{\text {optimum }}, \alpha_{\text {optimum }}, \beta_{\text {optimum }}\right)$ are calculated for each GGD distribution. The GGD that has the highest membership probability ( $p_{\text {optimum }}$ ) was selected as the pdf of the source and its parameters were used in the ICA algorithm.

\section{Simulation Phantom}

A simulation study was conducted to simulate DCE-MR images of a leaky phantom using a combination of finite element analysis (FEA) and classical description of MRI physics by means of Bloch equations.

Leakage Model (Finite Element Analysis): The Comsol Multiphysics (Comsol Inc., Burlington, MA, USA) finite element analysis (FEA) software was used to construct the simulated phantom that is shown in Fig. 1. This phantom is comprised of a grid of $10 \times 10$ leaky tubes that run in parallel through a cubic chamber of agar gel $(0.5 \%$ agar gel). The tubes have internal diameter of $200 \mu \mathrm{m}$, wall thickness of $30 \mu \mathrm{m}$ and center to center spacing of $300 \mu \mathrm{m}$. To model our experimental phantom experiments some of the tubes are removed to simulate the blocked or damaged tubes as shown in Fig. 1. The study simulated the passage of a bolus of contrast agent through the tubes and its leakage from the tubes into the agar gel over time. The spacing and diameter of the tubes were selected such that the vascular

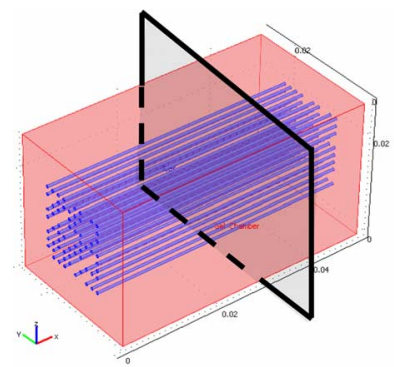

(a)

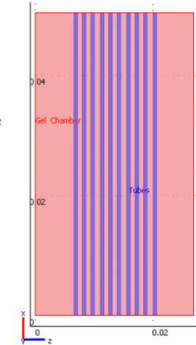

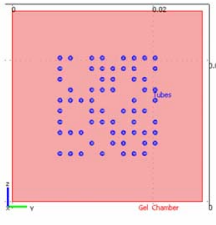

Fig. 1. a: 3-D view of the simulation phantom, it also shows the imaging plane that is located halfway through the phantom in $x$-direction and lies in the yz-plane. b: The xz plane showing the tubes are parallel in the gel. c: The view of the phantom from zy-plane that lies in the MR imaging plane.

fraction of the phantom is $3.8 \%$ so that it simulates the vascular volume fraction of a tumor tissue [27].

The 2-D DCE-MRI data was simulated for an imaging plane half way through the length of the chamber, transverse to the phantom as depicted in Fig. 1(a). A cross-section showing the orientation of the tubes in the imaging plane is shown in Fig. 1(c). As the contrast agent arrives in the transverse plane it diffuses into the surrounding gel. A range of different diffusion coefficient was assigned to the gel, the tubes and tube walls to account for variability of diffusion throughout the gel and generate a heterogeneous leakage apace. As such, the imaging plane was split into 2555 subdomains as shown in Fig. 2(a) with different diffusion coefficients. It was assumed that the diffusion coefficient of the gel had a uniform probability distribution with mean value of $2.08 \times 10^{-4} \mathrm{~mm}^{2} \cdot \mathrm{s}^{-1}$ [28] and standard deviation of $1 \times 10^{-5} \mathrm{~mm}^{2} \cdot \mathrm{s}^{-1}$. The subdomains that constructed the inside of the tubes were given the highest diffusion coefficients $\left(1 \times 10^{-3} \mathrm{~mm}^{2} \cdot \mathrm{s}^{-1}\right)$ as they were simulating flow of water and the subdomains corresponding to the walls of the tubes were assigned the lowest diffusion coefficients $\left(2 \times 10^{-5} \pm 10^{-6} \mathrm{~mm}^{2} \cdot \mathrm{s}^{-1}\right)$. The subdomains around one of the tubes showing the inner circle of the tube, the tube wall and the surrounding gel subdomains are also illustrated in Fig. 1(a). The simulation was performed for $6.48 \mathrm{~min}$ with a temporal resolution of $3.3 \mathrm{~s}$ which was chosen according to our experimental study. The flow rate and contrast concentration of the simulated bolus that was applied to the tubes at the $\mathrm{x}=0$ plane was selected such that the concentration-time curve of the tubes in the imaging plane was the same as our experimental studies. The contrast concentration distribution at time $2.5 \mathrm{~min}$ after injection of the contrast agent is shown in Fig. 2(b) which shows the heterogeneous distribution of contrast agent in the phantom.

Dynamic Contrast Enhanced-MRI Simulation: It was assumed that water was flowing through the tubes and the bolus of Gadolinium (Gd)-DTPA contrast agent is added to the flowing water. The geometry and contrast concentration of the tubes and the agar gel that were calculated in the FEA were fed to the developed MRI simulation software, and 120 frames were simulated to generate the DCE-MRI dataset. This section provides an overview of the 2-D MRI simulator that solved the Bloch equation at each voxel. It was developed based on the SIMRI project which was developed to simulate MR images 


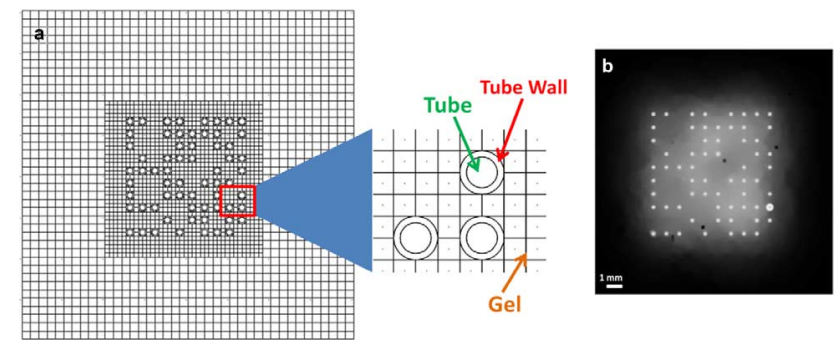

Fig. 2. a: FE subdomains of the phantom in the imaging plane. There are 2555 subdomains in this plane with different diffusion coefficients. As shown in the enlarged region of the phantom, the tubes, their walls and their surrounding gel areas are separated and proper diffusion coefficients are assigned to the subdomains of each region. b: Contrast agent distribution in the imaging plane, at time $=2.5 \mathrm{~min}$ after injection of the contrast agent. This figure shows the heterogeneous distribution of the contrast agent in the phantom.

[29]. The developed simulator starts with assigning proton density $\rho$, longitudinal relaxation $T_{1}$, and transverse relaxation $T_{2}$ to each voxel; these parameters are necessary for computing local spin magnetization. It was assumed that each voxel is comprised of two isochromates [30] corresponding to the tube and gel compartments.

The proton density of each isochromate at each voxel was calculated by taking the percentage of the voxel that belonged to the tubes/gel, which was available from the FEA step, multiplied by the proton density of water/gel. The pre-contrast $T_{1}$ relaxations $\left(T_{10}\right)$ of water and $0.5 \%$ agar gel were set to be 3000 $\mathrm{ms}$ and $2100 \mathrm{~ms}$ respectively. The pre-contrast $T_{2}$ relaxations $\left(T_{20}\right)$ of the water and gel were set to $250 \mathrm{~ms}$ and $65 \mathrm{~ms}$, respectively, [29], [31]. The post-contrast $T_{1}$ and $T_{2}$ values were calculated using the following equations [32]:

$$
\begin{aligned}
& \frac{1}{T_{1}}=\frac{1}{T_{10}}+R_{1}[G d] \\
& \frac{1}{T_{2}}=\frac{1}{T_{20}}+R_{2}[G d]
\end{aligned}
$$

where $T_{10}$ and $T_{20}$ are the pre-contrast longitudinal and transverse relaxations, respectively, $R_{1}=4.5 \mathrm{~mm}^{-1} \cdot \mathrm{s}^{-1}$ and $R_{2}=$ $5.5 \mathrm{~mm}^{-1} \cdot \mathrm{s}^{-1}$ are the longitudinal and transverse relaxivities of contrast agent respectively and [Gd] is the contrast concentration of water/gel that was calculated in the FEA step for each voxel at each frame of the dynamic sequence. The use of two isochromates in each voxel facilitates a realistic simulation of the cases where there are two different materials in the voxel. It also allows for simulation of intra-voxel de-phasing of spins in the voxel.

The dynamic contrast-enhanced image simulation was performed assuming a $\mathrm{B}_{0}$ magnetic field of $1.5 \mathrm{~T}$ with 1 ppm inhomogeneity using a single coil RF pulse. 2-D spoiled gradient recalled (SPGR) sequence of 120 frames (temporal resolution $=3.3 \mathrm{~s}$ ) were simulated. Other imaging parameters of the MRI simulation were: $\mathrm{TR}=12.5 \mathrm{~ms}, \mathrm{TE}=$ $2.9 \mathrm{~ms}, \mathrm{FA}$ (flip angle) $=20$, band width $(\mathrm{BW})=15.63 \mathrm{kHz}$, $\mathrm{Nx} / \mathrm{Ny}=128 / 128$, field of view $($ FOV $)=19.2 \mathrm{~mm}$, slice thickness $=5 \mathrm{~mm}$. Gaussian noise was added to the k-space data and its standard deviation was selected such that an SNR of 20 was achieved in image space.

The simulated DCE-MRI data was reconstructed at four different resolutions. The high resolution dataset had in-plane res- olution of $150 \mu \mathrm{m}$. In other datasets high frequency elements were removed and the low resolution datasets had in-plane resolutions of 300,600 , and $800 \mu \mathrm{m}$. A sample frame of each dataset at time $=2.5 \mathrm{~min}$ after injection of the contrast agent is shown in Fig. 3.

The magnitude of the MRI data is usually used in PK analysis of tumors. Thus, the performance of the proposed adaptive complex ICA (AC-ICA) intravascular signal calculation technique was compared to the case in which ICA was applied to the magnitude of the MRI data (magnitude ICA or Mag-ICA). The use of DCE-MRI data with different resolutions was also used to assess the robustness of the AC-ICA technique in separating intravascular signal, particularly in low resolutions that are more commonly encountered in clinical trials.

Since ICA is a stochastic process, it is important to assess the reproducibility of the intravascular time-intensity curves. Therefore, to assess the reproducibility of the AC-ICA and Mag-ICA results, the DCE-MRI dataset at each resolution was generated five times. Although these datasets were generated with the same imaging, geometry and physical parameters, they differed in distribution of $\mathrm{B}_{0}$ inhomogeneity that was added to the main magnetic field as a random Gaussian signal (1 ppm inhomogeneity) and the Gaussian noise $(\mathrm{SNR}=20)$ that was added to the data. The Gaussian noise was added to the simulated k-space data where standard deviation of noise was selected such that the SNR in image space was $20(\mathrm{SNR}=20)$.

\section{E. Tissue Mimicking Phantom}

A physical phantom, similar to the simulation phantom, was constructed that consisted of a chamber of agar gel $(0.5 \mathrm{wt} \%$, Sigma-Aldrich Canada Ltd., Oakville, ON, Canada) used as tissue mimicking material and a grid of $10 \times 10$ dialysis tubing (Diapes PES-150, Baxter) representing the vascular component. These tubes that approximated the diameter of small arteries or large arterioles had inner diameter of $200 \mu \mathrm{m}$, wall thickness of $30 \mu \mathrm{m}$ and center to center spacing of $300 \mu \mathrm{m}$ and passed through the agar gel parallel to each other, as shown in Fig. 4(a). These porous tubes (pore size of 89-972 nm) allowed low-molecular weight contrast agent to freely diffuse from the tubes into the agar gel.

DCE-MR imaging was performed at a transverse plane in the middle of the phantom, as shown in Fig. 4(a). Water was flowing through the tubes and an MR image of the phantom at the imaging plane is shown in Fig. 4(b). The gadolinium (Gd)-DTPA contrast agent was injected to the water stream as a bolus. Once the bolus reached the chamber that contained the gel, it was capable of leaking from the tubes into the gel. The flow of water was kept at a constant rate of $0.047 \mathrm{ml} / \mathrm{s}$ which translates into a flow velocity within arteriole's physiological range [33]. In order to measure the contrast agent concentration inside the tubes, the outflow line of the flow was oriented such that it passed through the imaging plane as shown in Fig. 4(b) and (c). The MR signal of this outflow line was used as the actual tubes' signal (with a delay of $9.7 \mathrm{~s}$ ) and was used to validate the calculated intravascular time-intensity curve.

Dynamic contrast-enhanced imaging was performed using a 2-D fast spoiled gradient recalled (fSPGR) sequence with the following imaging parameters: $\mathrm{TR}=12.5 \mathrm{~ms}$, $\mathrm{TE}=2.9 \mathrm{~ms}$, flip angle $=20, \mathrm{BW}=15.63 \mathrm{kHz}, \mathrm{Nx} / \mathrm{Ny} / \mathrm{NEX}=$ 

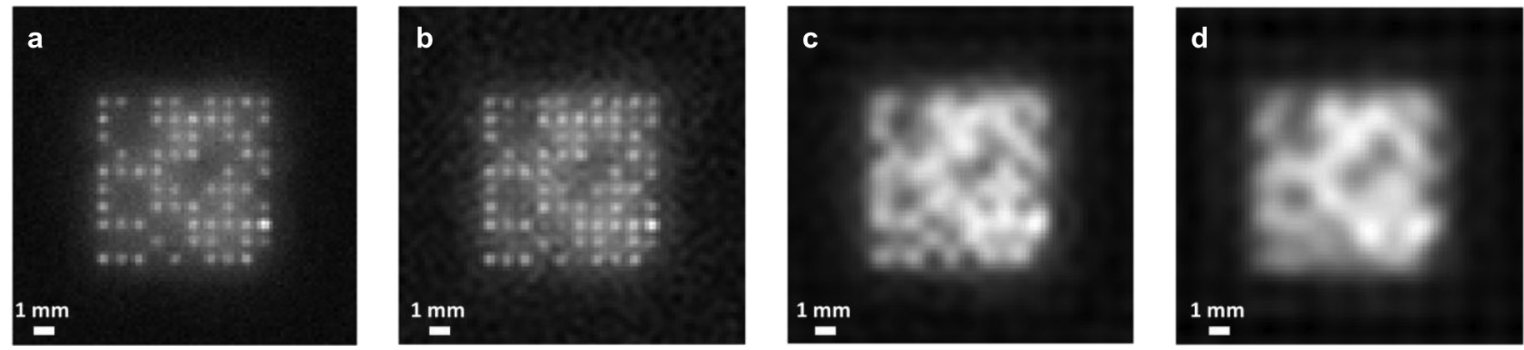

Fig. 3. Sample frame of each of the of the four datasets at time $=2.5 \mathrm{~min}$ after injection of the contrast agent, in-plane resolution of a: $150 \mu \mathrm{m} ; \mathrm{b}: 300 \mu \mathrm{m}$; c: $600 \mu \mathrm{m}$; and d: $800 \mu \mathrm{m}$.
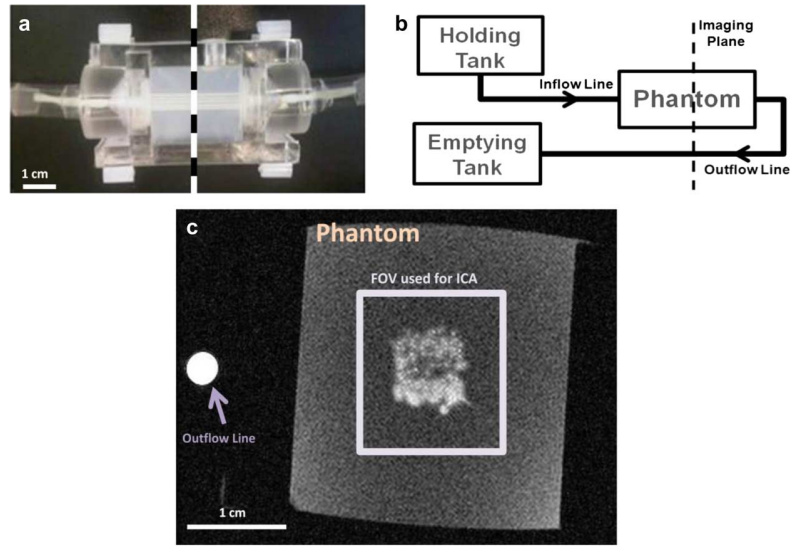

Fig. 4. a: Photograph of the physical phantom used in this study. Imaging was performed across the dashed line in the middle of the phantom, in a plane perpendicular to view that is shown in the photo. b: Schematic of the tissue mimicking phantom set up. Water was flowing at a constant rate $(0.047 \mathrm{ml} / \mathrm{s})$ from the holding tank to the emptying tank. The contrast agent was injected into the inflow line and the phantom was imaged at the imaging plane that included the phantom and the outflow line as shown in the figure. c: Sample MR image of the full imaging field of view (FOV) at time $=1.8 \mathrm{~min}$ after injection of the contrast agent that shows the outflow line, the entire phantom at imaging plane and the FOV around the tubes that is used for ICA analysis.

$256 / 256 / 1, \mathrm{FOV}=45 \mathrm{~mm}$, slice thickness $=5 \mathrm{~mm}$. A total of 120 images were acquired over about 6.48 min with a temporal resolution of $3.3 \mathrm{~s}$ and no delay between acquisitions. As in the simulation study, the data was reconstructed in different resolutions to assess the performance and robustness of the AC-ICA and compare it to the Mag-ICA and actual time-intensity curve of the tubes (measured at the outflow line). The image reconstruction was performed at five different in-plane resolutions of $170,225,340,450$, and $680 \mu \mathrm{m}$. A sample frame of each dataset at time $=1.8 \mathrm{~min}$ after injection of the contrast agent, is shown in Fig. 5(a)-(e).

In order to assess the reproducibility of the intravascular timeintensity curvs for both AC-ICA and Mag-ICA algorithm, two phantoms with identical specifications were built and DCE-MRI imaging with the same imaging parameters was performed on both phantoms.

\section{RESULTS}

\section{A. Simulation Phantom Study}

The magnitude ICA (Mag-ICA) method introduced in [15], [17] as well as the adaptive complex ICA (AC-ICA) for intravascular time-intensity curve calculation were applied to all simulated datasets (four different resolutions) and the signal from inside the tubes (intravascular signal) was extracted. Dimensionality reduction was performed on each dataset through singular value decomposition (SVD) of the covariance matrix of the data and only the eigenvalues that were larger than the $0.1 \%$ of the largest eigenvalue were kept which translated into keeping approximately $99.9 \%$ of the information in the dataset. The number of the eigenvalues that were kept for analysis ranged between 5 and 8 which is the maximum number of IC's that could be estimated. In all cases, all IC's were estimated and the IC that corresponded to the tubes' signal was selected heuristically. The IC that had a uniform pre-contrast uptake followed by a rapid contrast uptake and also a rapid wash-out of the contrast agent to less than $60 \%$ of the peak signal intensity within 3 min of injection of the bolus was selected as the tubes' signal.

Fig. 6 shows the results of applying ICA to the magnitude of the simulated DCE-MR images (Mag-ICA). The intravascular component images for all datasets (four different resolutions) are shown in Fig. 6(a)-(d). Fig. 6(e) shows the intravascular time-intensity curve of the four datasets. These curves represent the average signal intensity over time of all the voxels that are separated by ICA as the intravascular space (tubes). Fig. 6(e) also shows the actual intravascular time-intensity curve which was calculate by averaging the signal across the outflow line [shown in Fig. 4(c)] over time as well as the raw data curve calculate by averaging the signal across the raw MRI images over time.

The time-intensity curves are normalized with respect to their maximum and the baseline signal intensity is set to zero in order to enable comparison. In Fig. 7 the results of applying AC-ICA to the simulated complex-valued DCE-MRI data are illustrated. The extracted IC images of tubes for all four resolutions are shown in Fig. 7(a)-(d). The time-intensity curves of the tubes for all four simulated datasets as well as the actual time-intensity curve of the tubes and the raw data curve are shown in Fig. 7(e). The time-intensity curves are normalized with respect to their maximum and the pre-contrast signal intensity is set to zero in order to enable comparison.

The IC images extracted using ACICA had complex values and thus each voxel is represented with a $2-\mathrm{D}$ vector (magnitude and phase) while each voxel in the images shown in Fig. 7(a)-(d) is represented with a 1-D value (image signal intensity). This conversion was required for visualization purposes and was performed by assigning negative sign to the magnitude of the values of the voxels that had $-\pi / 2 \leq$ phase $<$ 

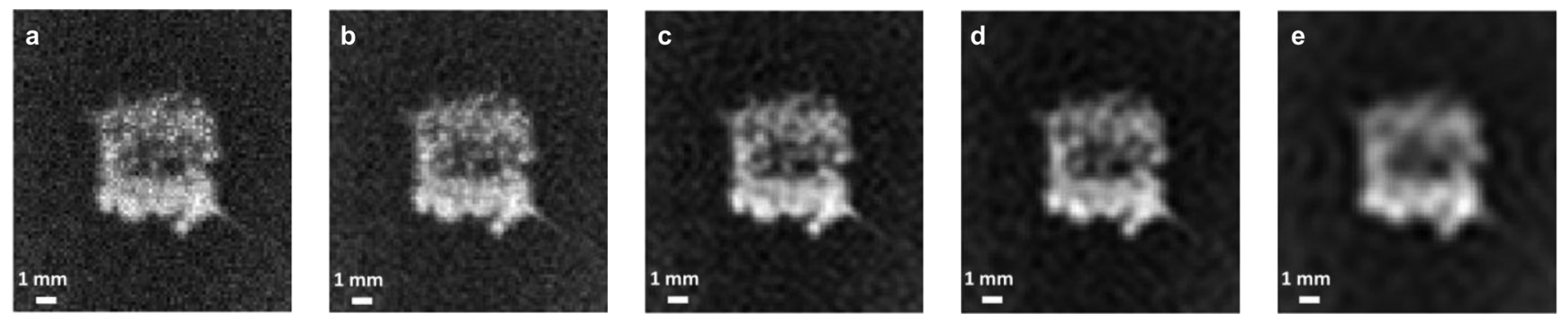

Fig. 5. Sample frame of each of the five experimental DCE-MRI datasets of the phantom at time $=1.8$ min after injection of the contrast agent, with in-plane resolutions of: a: $170 \mu \mathrm{m}$; b: $225 \mu \mathrm{m}$; c: $340 \mu \mathrm{m}$; d: $450 \mu \mathrm{m}$; and e: $680 \mu \mathrm{m}$.
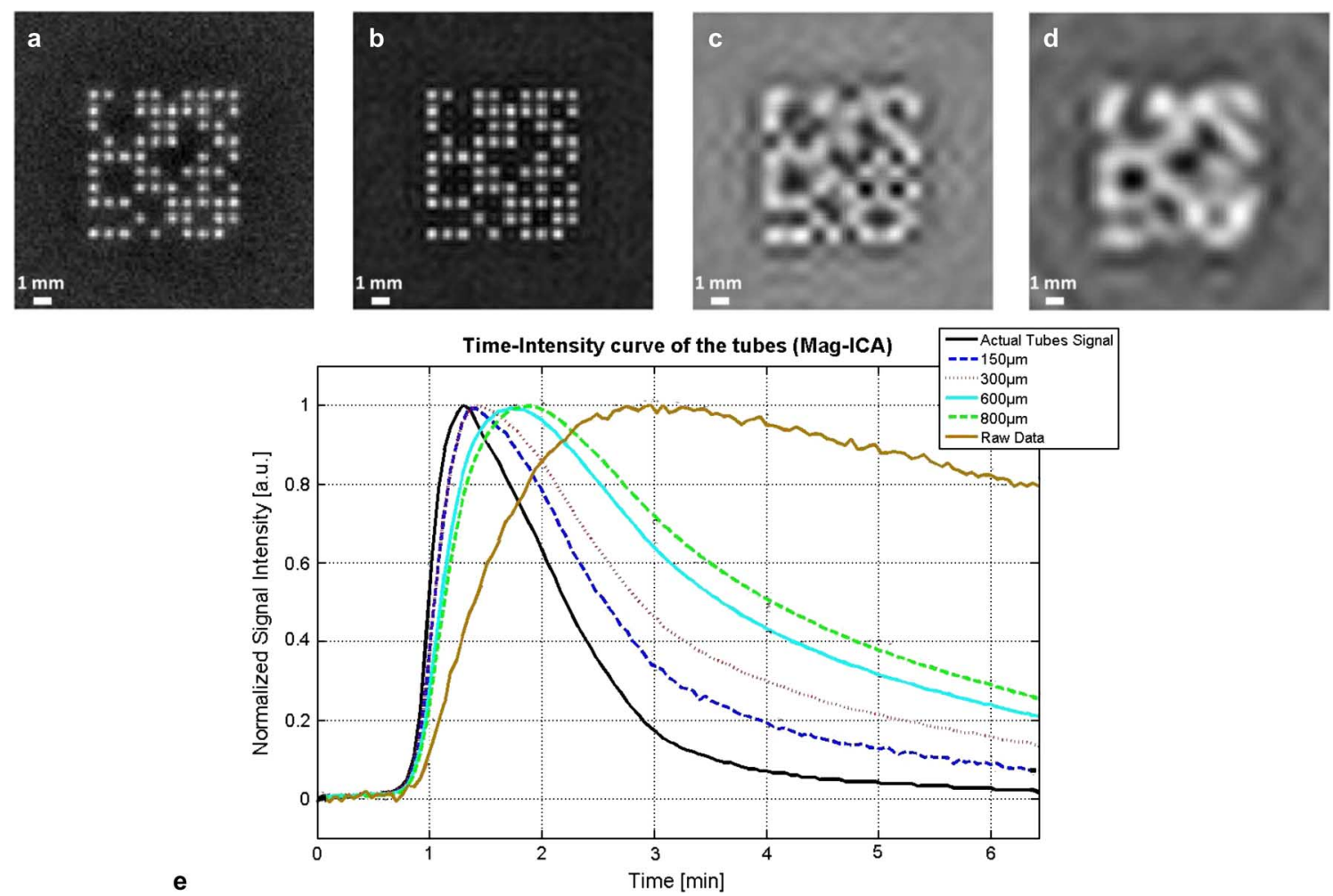

Fig. 6. The separated tubes image and the time-intensity curve of the tubes calculated using Mag-ICA for each of the four simulated DCE-MRI datasets. Tubes images for datasets with in-plane resolutions of a: $150 \mu \mathrm{m}$; b: $300 \mu \mathrm{m}$; $: 600 \mu \mathrm{m} ; \mathrm{d}: 800 \mu \mathrm{m}$. e: Calculated time-intensity curves of the tubes corresponding to the four simulated datasets, the Actual time-intensity curve of the tubes and the curve corresponding to the mean across the entire raw (not analyzed) images over time (raw data).

$\pi / 2$ and assigning positive sign to the magnitude of the values of the voxel with phase $\geq \pi / 2$ or phase $<-\pi / 2$. This method of visualizing the images provides consistent results with the Mag-ICA analysis where we had both negative and positive signal intensity values.

For each in-plane resolution, five DCE-MRI data sets of the phantom were simulated with SNR $=20$. The AC-ICA and Mag-ICA algorithms were applied to all datasets. Table I reports the root mean square error (RMSE) between the estimated timeintensity curves of the tubes obtained using each algorithm and the actual curve for all four datasets. Table I also reports the correlation coefficient between the estimated and actual timeintensity curves of the tubes for both algorithms.

\section{B. Experimental Phantom Study}

The adaptive complex ICA and magnitude ICA were also applied to DCE-MRI images of the experimental tissue mimicking phantom. Similar to the simulation study, dimensionality reductions was performed first, where eigenvalues that were larger that $0.1 \%$ of the largest eigenvalue were kept. The phantom data was reconstructed in five different in-plane resolutions and both algorithms were applied to all five datasets. Fig. 8 shows the results of applying the Mag-ICA algorithm to the experimental DCE-MRI data. The IC images corresponding to the tubes' signal of the five datasets are shown in Fig. 8(a)-(e). The time-intensity curves of the five datasets as well as the actual time-intensity curve of the tubes that was measured at the outflow line of the phantom and the raw data curve calculate by averaging the signal across the raw MRI images over time are shown in Fig. 8(f). The baseline values of the time-intensity curves of all datasets were set to zero and they were normalized with respect to their maximum values.

Fig. 9 shows the results of applying the proposed AC-ICA algorithm to the DCE-MRI data of the experimental phantom 

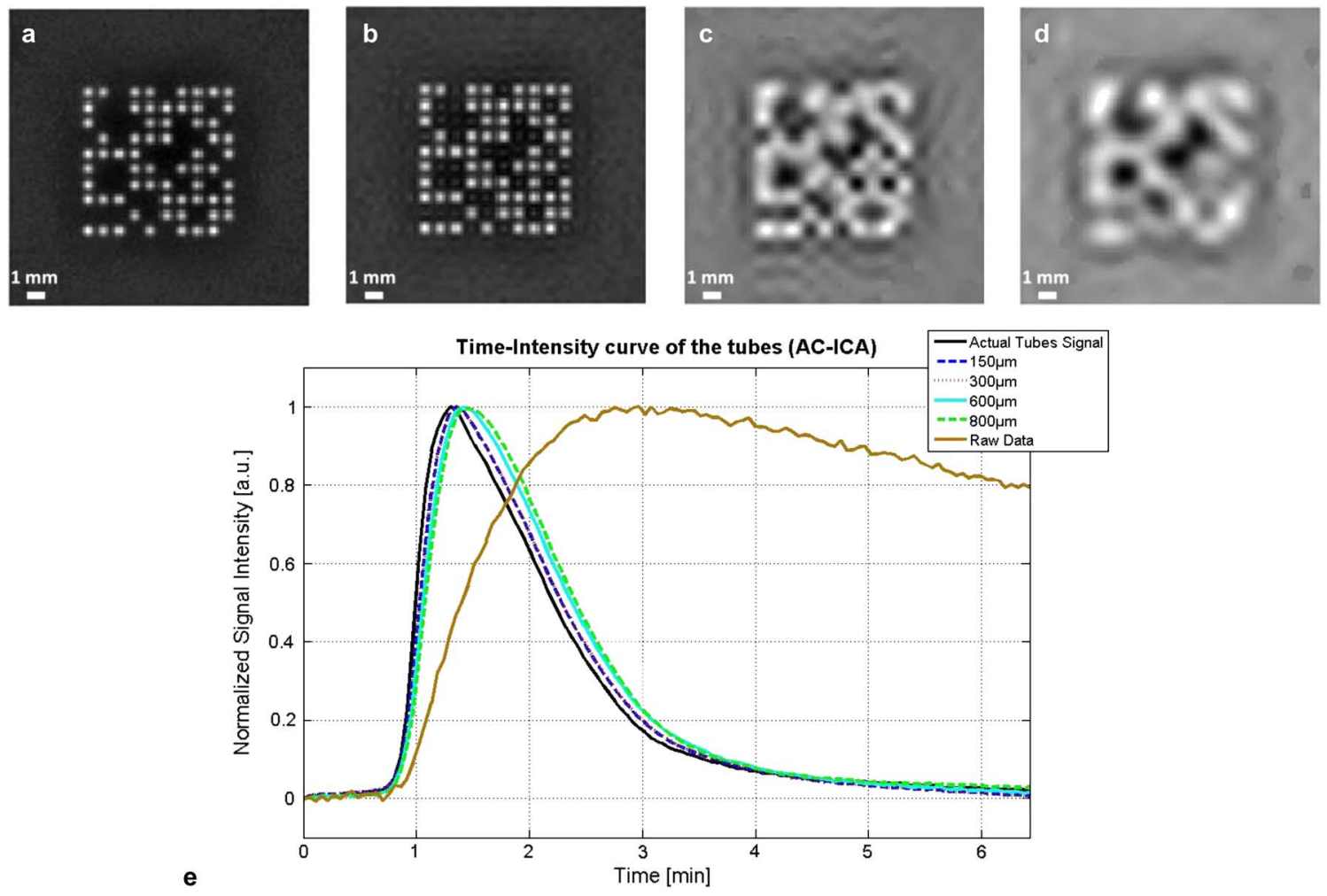

Fig. 7. The separated tubes image and the time-intensity curve of the tubes calculated using AC-ICA for each of the four simulated DCE-MRI datasets. Tubes images for datasets with in-plane resolutions of a: $150 \mu \mathrm{m}$; b: $300 \mu \mathrm{m} ; \mathrm{c}: 600 \mu \mathrm{m} ; \mathrm{d}: 800 \mu \mathrm{m}$. e: Calculated time-intensity curves of the tubes corresponding to the four simulated datasets, the Actual time-intensity curve of the tubes and the curve corresponding to the mean across the entire raw (not analyzed) images over time (raw data).

TABLE I

Root MEAN SQuare ERror (RMSE) AND CORRELATION COEFFICIENT BetWeEn the Estimated Tubes' TIME-INTENSITY CuRVES AND THE ACtual CuRve for Four Dataset IN Both ICA Algorithms (RMSE and Correlation Coefficients are Calculated After SetTing the Baseline VAlues of the CURVes to Zero AND NoRmalizing THEM)

\begin{tabular}{ccccc}
\hline $\begin{array}{c}\text { In-plane } \\
\text { Resolution }\end{array}$ & $150 \mu \mathrm{m}$ & $300 \mu \mathrm{m}$ & $600 \mu \mathrm{m}$ & $\mathbf{8 0 0 \mu \mathrm { m }}$ \\
\hline \multicolumn{5}{c}{ Root Mean Square Error (RMSE) } \\
\hline \multirow{2}{*}{ AC-ICA } & $0.034 \pm$ & $0.035 \pm$ & $0.065 \pm$ & $0.077 \pm$ \\
& 0.006 & 0.004 & 0.018 & 0.013 \\
& $0.11 \pm$ & $0.19 \pm$ & $0.3 \pm$ & $0.36 \pm$ \\
Mag-ICA & 0.03 & 0.01 & 0.08 & 0.04 \\
\hline & \multicolumn{5}{c}{ Correlation Coefficient } \\
\hline \multirow{2}{*}{ AC-ICA } & $0.994 \pm$ & $0.993 \pm$ & $0.981 \pm$ \\
& 0.002 & 0.001 & 0.007 & $0.071 \pm$ \\
& $0.964 \pm$ & $0.924 \pm$ & $0.768 \pm$ & $0.685 \pm$ \\
Mag-ICA & 0.016 & 0.005 & 0.087 & 0.06 \\
\hline
\end{tabular}

for all five different in-plane resolutions. The separated tubes' images of the five datasets are shown in Fig. 9(a)-(e). The corresponding time-intensity curves of the tubes in these datasets as well as the actual time-intensity curve of the tubes measured at the outflow curve of the phantom and the raw data curve are shown in Fig. 9(f). The baseline values of the time-intensity curves of all datasets were set to zero and they were normalized with respect to their maximum values.

Two experimental phantoms were built and DCE-MRI imaging was performed on both phantoms to assess the reproducibility of the results for both intravascular time-intensity curve calculation algorithms. Table II reports the root mean square error (RMSE) between the estimated and the actual time-intensity curves of the tubes (outflow) for all five datasets of both phantoms. This table also reports the correlation coefficient between the estimated and actual time-intensity curves of the tubes for both algorithms in all five in-plane resolutions.

\section{Discussions}

Measurements of tumor size and serum markers are not efficient criteria in assessing tumor response to therapy particularly in anti-angiogenic therapies. Pharmacokinetic modeling of DCE-MR images of a tumor is one of the approaches used to assess its therapeutic response. A fundamental step in PK modeling that is common amongst most models is determining the intravascular contrast agent concentration which is approximated using an arterial input function (AIF). Mehrabian et al. [15], [17] developed an ICA-based method to measure and separate signal that is generated in the tumor vasculature. Although the output of MR imaging is complex-valued, only the magnitude data was used (as is common in most DCE-MRI studies) and the phase information was not utilized in the study. This introduced a fundamental challenge in ICA analysis as the linear 

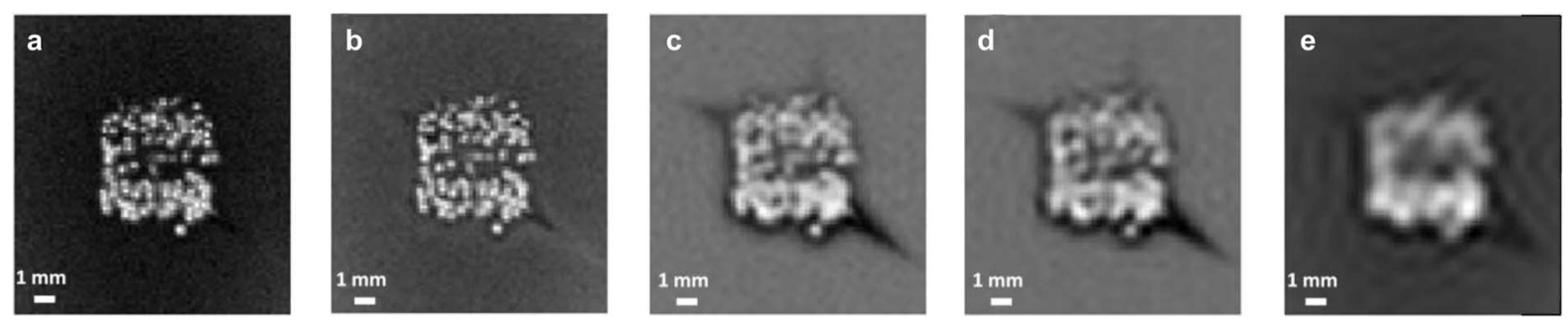

Time-Intensity curve of the tubes (Mag-ICA)

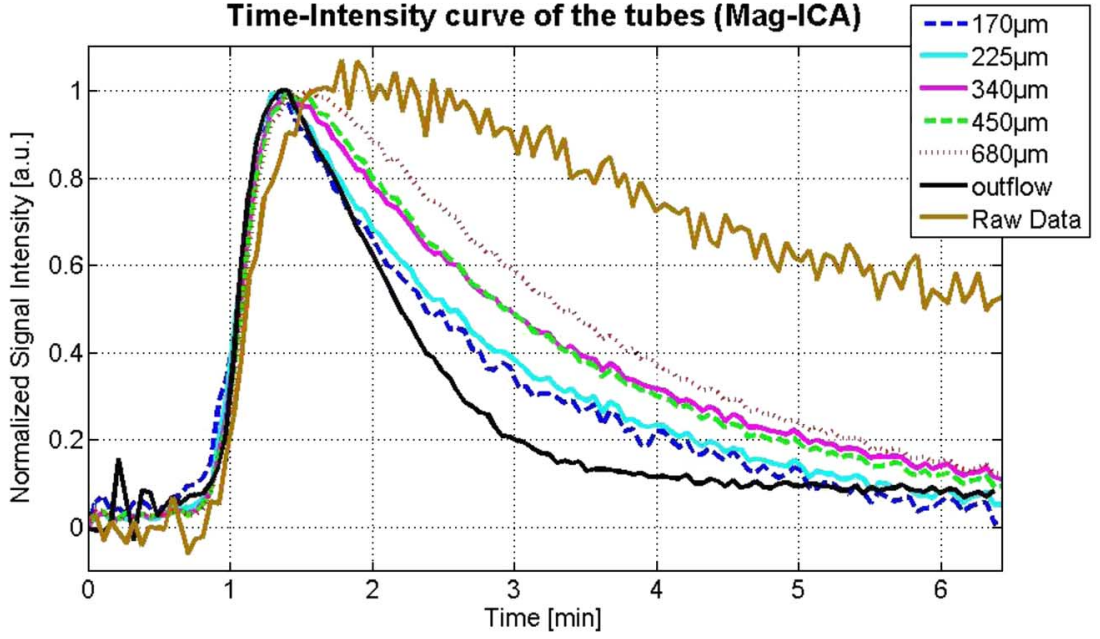

Fig. 8. The separated tubes image and the time-intensity curve of the tubes calculated using Mag-ICA for each of the five experimental DCE-MRI datasets of the tissue mimicking phantom. Tubes images for datasets with in-plane resolutions of a: $170 \mu \mathrm{m}$; b: $225 \mu \mathrm{m}$; c: $340 \mu \mathrm{m}$; $: 450 \mu \mathrm{m}$; and e: $680 \mu \mathrm{m}$. e) Calculated time-intensity curves of the tubes corresponding to the five experimental datasets, the Actual time-intensity curve of the tubes (outflow) and the curve corresponding to the mean across the entire raw (not analyzed) images over time (raw data).

mixture assumption of ICA was violated. This problem was addressed by using short echo time (TE) and minimizing intravoxel de-phasing [15], [17]. The separated intravascular image and time-intensity curves were validated against intravascular contrast enhanced ultrasound measurements in both phantoms and animal models.

An adaptive complex ICA (AC-ICA) method was introduced in this study that used the complex-valued MRI data for ICA and also used an adaptive cost function for ICA. The adaptive cost function was determined by assuming the probability distribution of MRI data takes the form of a mixture of generalized Gaussian distributions (GGD) whose parameters were estimated using an expectation maximization (EM) approach. Simulated and experimental phantoms were constructed and their theoretical and experimental DCE-MRI data were acquired respectively. Each phantom was comprised of a grid of leaky tubes that represented the tumor vasculature embedded in a chamber of agar gel that represented the extravascular extracellular space of the tumor.

The AC-ICA method was applied to the DCE-MRI data of simulated and experimental phantoms and the tubes' time-intensity signal was estimated. These results were compared with the results of calculating time-intensity curves of the tubes using the Mag-ICA method that was introduced in [15], [17] to highlight the better performance of the AC-ICA. Both experimental and simulation data where reconstructed in different resolutions to assess the robustness of the method and its capability in separating signal of the tubes in low resolution data that are more common in clinical practice.
As shown in Figs. 6 and 7, and Table I, in simulation study both AC-ICA and Mag-ICA methods were capable of separating time-intensity curve of the tubes with high accuracy for high resolution data in both spatial and temporal domains. As the voxel size increased (lower resolution), the AC-ICA demonstrated higher accuracy and robustness compared to Mag-ICA in dealing with datasets with a wide range of in-plane resolutions. There were small differences between the time-intensity curves of the tubes calculated using AC-ICA for different resolutions while Mag-ICA curves changed significantly and its time-intensity curves were not good for the lower resolutions. Note that in lower resolutions although the tubes could not be visualized in the spatial domain, however; their time-intensity curve was calculated with good accuracy.

Similar results were obtained in the experimental DCE-MRI data as shown in Figs. 8 and 9 and reported in Table II. The tubes were separated accurately in both spatial and temporal domains for the high resolution data. As the pixel size increased it became more difficult to visualize the tube in the IC images such that in the two lowest resolutions it was impossible to see them separately from the leakage. However the time-intensity curve of the tubes was estimated with high accuracy for all resolutions in both AC-ICA and Mag-ICA methods. Similar to the simulation studies, the AC-ICA demonstrated better accuracy in dealing with lower resolution data and there were smaller differences between time-intensity curves of the tubes at varying resolutions compared to the Mag-ICA.

There was an aura in the experimental phantom images outside of the tubes which is due to fabrication artifact. A few of 

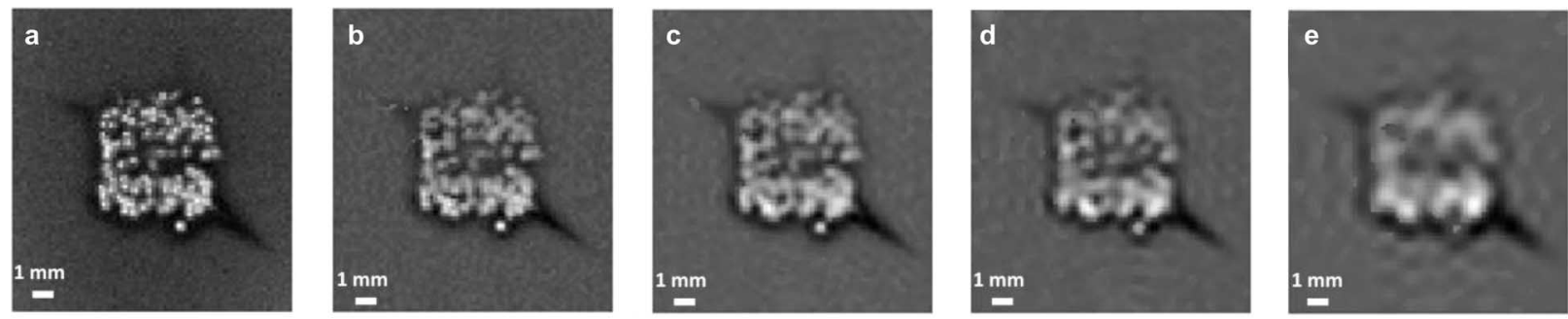

Time-Intensity curve of the tubes (AC-ICA)

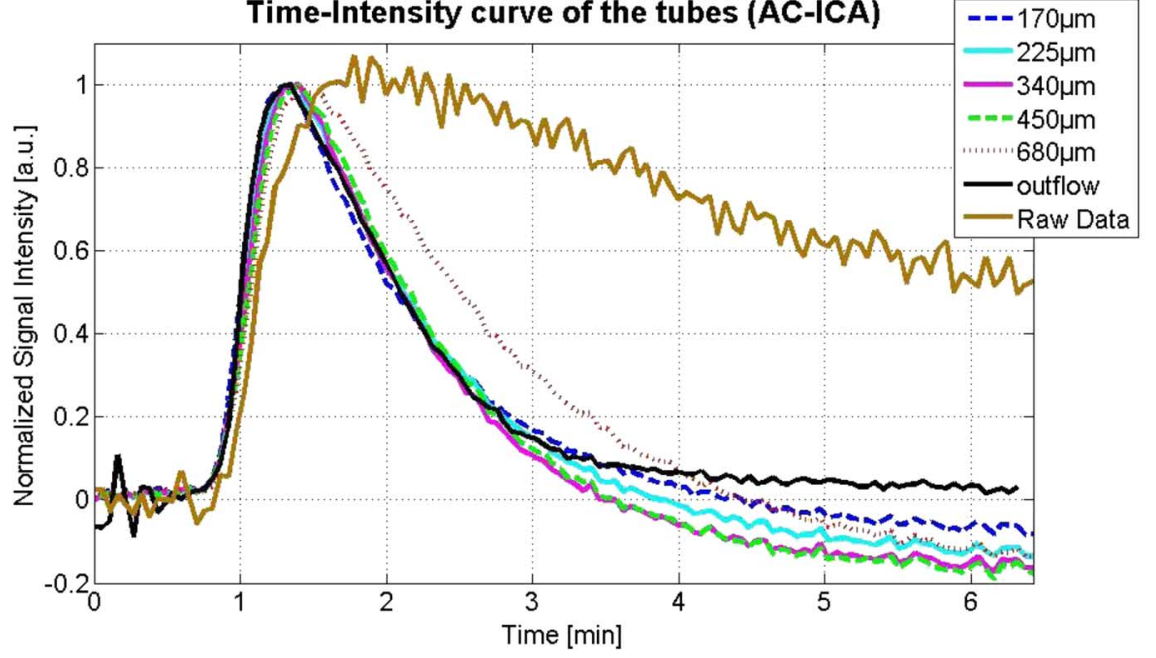

Fig. 9. The separated tubes image and the intravascular time-intensity curve of the tubes calculated using AC-ICA for each of the five experimental DCE-MRI datasets of the tissue mimicking phantom. Tubes images for datasets with in-plane resolutions of a) $170 \mu \mathrm{m}$; b) $225 \mu \mathrm{m}$; c) $340 \mu \mathrm{m}$; d) $450 \mu \mathrm{m}$; and e) $680 \mu \mathrm{m}$. e) Calculated time-intensity curves of the tubes corresponding to the five experimental datasets, the Actual time-intensity curve of the tubes (outflow) and the curve corresponding to the mean across the entire raw (not analyzed) images over time (raw data).

TABLE II

RMSE AND THE CORRELATION COEFFICIENT BETWEEN THE EsTIMATED AND ACTUAL TIME-INTENSiTy CURVES OF THE TUBES (OUTFLOW) FOR ALL Five Datasets of Both Phantoms For Mag-ICA AND AC-ICA (RMSE and Correlation Coefficients are Calculated After Setting the BASELINE OF THE CURVES TO ZERO AND NORMALIZING THEM)

\begin{tabular}{|c|c|c|c|c|c|}
\hline $\begin{array}{l}\text { In-plane } \\
\text { Resolution }\end{array}$ & $170 \mu \mathrm{m}$ & $225 \mu \mathrm{m}$ & $340 \mu \mathrm{m}$ & $450 \mu \mathrm{m}$ & $680 \mu \mathrm{m}$ \\
\hline \multicolumn{6}{|c|}{ Root Mean Square Error (RMSE) } \\
\hline AC-ICA & $\begin{array}{c}0.038 \pm \\
0.001\end{array}$ & $\begin{array}{c}0.054 \pm \\
0.002\end{array}$ & $\begin{array}{c}0.079 \pm \\
0.001\end{array}$ & $\begin{array}{c}0.084 \pm \\
0.001\end{array}$ & $\begin{array}{c}0.129 \pm \\
0.011\end{array}$ \\
\hline Mag-ICA & $\begin{array}{c}0.089 \pm \\
0.003\end{array}$ & $\begin{array}{c}0.124 \pm \\
0.026\end{array}$ & $\begin{array}{c}0.186 \pm \\
0.009\end{array}$ & $\begin{array}{c}0.192 \pm \\
0.018\end{array}$ & $\begin{array}{c}0.248 \pm \\
0.004\end{array}$ \\
\hline \multicolumn{6}{|c|}{ Correlation Coefficient } \\
\hline AC-ICA & $\begin{array}{c}0.994 \pm \\
0.001\end{array}$ & $\begin{array}{c}0.987 \pm \\
0.001\end{array}$ & $\begin{array}{c}0.993 \pm \\
0.002\end{array}$ & $\begin{array}{c}0.975 \pm \\
0.002\end{array}$ & $\begin{array}{c}0.938 \pm \\
0.011\end{array}$ \\
\hline Mag-ICA & $\begin{array}{l}0.97 \pm \\
0.014\end{array}$ & $\begin{array}{l}0.95 \pm \\
0.017\end{array}$ & $\begin{array}{c}0.915 \pm \\
0.005\end{array}$ & $\begin{array}{c}0.908 \pm \\
0.014\end{array}$ & $\begin{array}{c}0.854 \pm \\
0.004 \\
\end{array}$ \\
\hline
\end{tabular}

the tubes were broken during the phantom construction process and the contrast agent was capable of leaving these broken tubes more easily. The results show that this aura is smaller in the tubes image of the ACICA analysis (particularly in the high-resolution images) compared to the Mag-ICA results which shows that ACICA is capable of separating the intravascular space more accurately.

There are two reasons for superior performance of AC-ICA: 1) the ICA cost function is adapted at every iteration to match the probability distribution of the tubes' signal, 2) unlike the Mag-ICA, the linear mixture assumption of ICA is not violated in AC-ICA and thus intra-voxel de-phasing (spins inside each voxel are not necessarily in-phase) and partial volume effect do not play as significant role as they do in Mag-ICA.

Moreover, in the experimental study the signal intensity of the tubes dropped below its baseline value. This effect is currently under investigation, however it could be associated with the effects of the contrast agent that leaked to the EES on the $T_{2}^{*}$ value of the voxels inside the tubes. It could also be associated with the effects of the contrast agent that diffused too far from the tubes, such that it could not return to the tubes and could not be washed out.

In this study we developed an adaptive complex ICA-based method to calculate and separate the intravascular signal in DCE-MRI datasets. However, in PK analysis of tumor tissues, this signal intensity has to be converted into contrast agent concentration. This is not a trivial step for the results of ICA-based method and requires further investigation as there is an arbitrary scaling of the ICA results.

It was shown that although the tubes were not visible in the IC images of low resolution datasets (both simulation and experiment), the intravascular time-intensity curves in low resolution datasets are very close to the high resolution ones. This 
demonstrates that ICA-based calculation of the intravascular time-intensity curve has the potential to be used in clinical studies, where the resolution of DCE-MRI data is very low. The AC-ICA method provided more accurate results compared to Mag-ICA which suggests that complex-valued (magnitude and phase) DCE-MRI data should be used to calculate the intravascular time-intensity curve in the tumor. This could lead to more accurate PK analysis and better understanding of the tumor response to therapy.

\section{REFERENCES}

[1] C. H. Lee, L. Braga, R. O. de Campos, and R. C. Semelka, "Hepatic tumor response evaluation by MRI," NMR Biomed., vol. 24, no. 6, pp. 721-733, 2011.

[2] W. A. Weber, "Assessing tumor response to therapy," J. Nucl. Med., vol. 50, pp. 1S-10S, 2009, Suppl. 1.

[3] J. J. Smith, A. G. Sorensen, and J. H. Thrall, "Biomarkers in imaging: Realizing radiology's future," Radiology, vol. 227, no. 3, pp. 633-638, 2003.

[4] M. Kanematsu, S. Osada, N. Amaoka, S. Goshima, H. Kondo, H. Kato, H. Nishibori, R. Yokoyama, H. Hoshi, and N. Moriyama, "Expression of vascular endothelial growth factor in hepatocellular carcinoma and the surrounding liver and correlation with MRI findings," Am. J. Roentgenol., vol. 184, no. 3, pp. 832-841, 2005.

[5] M. O. Leach, K. M. Brindle, J. L. Evelhoch, J. R. Griffiths, M. R. Horsman, A. Jackson, G. Jayson, I. R. Judson, M. V. Knopp, R. J. Maxwell, D. McIntyre, A. R. Padhani, P. Price, R. Rathbone, G. Rustin, P. S. Tofts, G. M. Tozer, W. Vennart, J. C. Waterton, S. R. Williams, and P. Workman, "Assessment of antiangiogenic and antivascular therapeutics using MRI: Recommendations for appropriate methodology for clinical trials," Br. J. Radiol., vol. 76, pp. S87-S91, 2003.

[6] J. H. Naish, L. E. Kershaw, D. L. Buckley, A. Jackson, J. C. Waterton, and G. J. M. Parker, "Modeling of contrast agent kinetics in the lung using T(1)-weighted dynamic contrast-enhanced MRI," Magn. Reson. Med., vol. 61, no. 6, pp. 1507-1514, Jun. 2009.

[7] P. S. Tofts and A. G. Kermode, "Measurement of the blood-brain-barrier permeability and leakage space using dynamic MR imaging. 1. Fundamental-concepts," Magn. Reson. Med., vol. 17, no. 2, pp. 357-367, 1991.

[8] H. L. M. Cheng, "Investigation and optimization of parameter accuracy in dynamic contrast-enhanced MRI," J. Magn. Reson. Imag., vol. 28, no. 3, pp. 736-743, 2008.

[9] A. E. Hansen, H. Pedersen, E. Rostrup, and H. B. W. Larsson, "Partial Volume Effect (PVE) on the Arterial Input Function (AIF) in T-1-weighted perfusion imaging and limitations of the multiplicative rescaling approach," Magn. Reson. Med., vol. 62, no. 4, pp. $1055-1059,2009$.

[10] D. M. McGrath, D. P. Bradley, J. L. Tessier, T. Lacey, C. J. Taylor, and G. J. M. Parker, "Comparison of model-based arterial input functions for dynamic contrast-enhanced MRI in tumor bearing rats," Magn. Reson. Med., vol. 61, no. 5, pp. 1173-1184, 2009

[11] R. Lawaczeck, G. Jost, and H. Pietsch, "Pharmacokinetics of contrast media in humans model with circulation, distribution, and renal excretion," Invest. Radiol., vol. 46, no. 9, pp. 576-585, Sep. 2011.

[12] D. A. Kovar, M. Lewis, and G. S. Karczmar, "A new method for imaging perfusion and contrast extraction fraction: Input functions derived from reference tissues," J. Magn. Reson. Imag., vol. 8, no. 5, pp. 1126-1134, 1998.

[13] A. Shukla-Dave, N. Lee, H. Stambuk, Y. Wang, W. Huang, H. T. Thaler, S. G. Patel, J. P. Shah, and J. A. Koutcher, "Average arterial input function for quantitative dynamic contrast enhanced magnetic resonance imaging of neck nodal metastases," BMC Med. Phys., vol. 9 , p. 4,2009
[14] X. Fan, C. R. Haney, D. Mustafi, C. Yang, M. Zamora, E. J. Markiewicz, and G. S. Karczmar, "Use of a reference tissue and blood vessel to measure the arterial input function in DCEMRI," Magn. Reson. Med., vol. 64, no. 6, pp. 1821-1826, Dec. 2010.

[15] H. Mehrabian, C. Chandrana, I. Pang, R. Chopra, and A. L. Martel, "Arterial input function calculation in dynamic contrast-enhanced MRI: An in vivo validation study using co-registered contrast-enhanced ultrasound imaging," Eur. Radiol., vol. 22, no. 8, pp. $1735-1747,2012$.

[16] J. G. Korporaal, M. Van Vulpen, C. A. T. Van Den Berg, and U. A. Van Der Heide, "Tracer kinetic model selection for dynamic contrastenhanced computed tomography imaging of prostate cancer," Invest. Radiol., vol. 47, no. 1, pp. 41-48, 2012.

[17] H. Mehrabian, I. Pang, C. Chandrana, R. Chopra, and A. L. Martel, "Automatic mask generation using independent component analysis in dynamic contrast enhanced-MRI," in Proc. Int. Symp. Biomed. Imag., 2011, pp. 1657-1661.

[18] P. Comon, "Independent component analysis, A new concept?," Signal Process., vol. 36, no. 3, pp. 287-314, 1994.

[19] A. Araujo and E. Giné, The Central Limit Theorem for Real and Banach Valued Random Variables. NY: Wiley, 1980.

[20] M. Novey and T. Adali, "Complex ICA by negentropy maximization,' IEEE Trans. Neural Netw., vol. 19, no. 4, pp. 596-609, Apr. 2008.

[21] A. van den Bos, "Complex gradient and Hessian," in IEE Proc Vis. Image Signal Proc., 1994, vol. 141, no. 6, pp. 380-382.

[22] M. Novey and T. Adalt, "Adaptable nonlinearity for complex maximization of nongaussianity and a fixed-point algorithm," in Proc. 2006 16th IEEE Signal Process. Soc. Workshop Mach. Learn. Signal Process., 2007, pp. 79-84.

[23] O. Bernard, J. D'hooge, and D. Friboulet, "Statistical modeling of the radio-frequency signal in echocardiographic images based on generalized Gaussian distribution," in Proc. 3rd IEEE Int. Symp. Biomed. Imag.: From Nano to Macro, 2006, pp. 153-156.

[24] M. S. Davis, P. Bidigare, and D. Chang, "Statistical modeling and ML parameter estimation of complex SAR imagery," in Conf. Rec. - Asilomar Conf. Signals, Syst. Comput., 2007, pp. 500-502.

[25] D. Gonzalez-Jimenez, F. Perez-Gonzalez, P. Comesana-Alfaro, L. Perez-Freire, and J. L. Alba-Castro, "Modeling Gabor coefficients via generalized Gaussian distributions for face recognition," in Proc. IEEE Int. Conf. Image Process., 2007, vol. 4, pp. 485-488.

[26] W. Rudin, Real and Complex Analysis. New York: McGraw-Hill, 1987.

[27] A. Wall, T. Persigehl, P. Hauff, K. Licha, M. Schirner, S. Muller, A. von Wallbrunn, L. Matuszewski, W. Heindel, and C. Bremer, "Differentiation of angiogenic burden in human cancer xenografts using a perfusion-type optical contrast agent (SIDAG)," Breast Cancer Res., vol. $10,2008$.

[28] T. S. Koh, S. Hartono, C. H. Thng, T. K. H. Lim, L. Martarello, and Q. S. Ng, "In vivo measurement of gadolinium diffusivity by dynamic contrast-enhanced MRI: A preclinical study of human xenografts," Magn. Reson. Med., vol. 69, no. 1, pp. 269-276, 2013.

[29] H. Benoit-Cattin, G. Collewet, B. Belaroussi, H. Saint-Jalmes, and C. Odet, "The SIMRI project: A versatile and interactive MRI simulator," J. Magn. Reson., vol. 173, no. 1, pp. 97-115, 2005.

[30] P. Latta, M. L. H. Gruwel, V. Jellúš, and B. Tomanek, "Bloch simulations with intra-voxel spin dephasing," J. Magn. Reson., vol. 203, no. 1, pp. 44-51, 2010.

[31] L. Cochlin, A. Blamire, and P. Styles, "Dependence of T1 and T2 on high field strengths in doped agarose gels; facilitating selection of composition for specific T1/T2 at relevant field," Int. Soc. Magn. Reson. Med., p. 885, 2003.

[32] R. Hendrick and E. Haacke, "Basic physics of MR contrast agents and maximization of image-contrast," J. Magn. Reson. Imag., vol. 3, no. 1, pp. 137-148, 1993.

[33] W. A. Ritschel and G. L. Kearns, Handbook of Basic Pharmacokinetics-Including Clinical Applications. Washington, D.C.: Am. Pharmaceutical Assoc., 1999. 